\title{
IDENTIFYING THE ISOMORPHISM OF KINEMATIC CHAINS
}

\author{
Krystyna ROMANIAK* \\ *Faculty of Architecture, Cracow University of Technology, ul. Warszawska 24, 31-155 Kraków, Poland \\ krystynaromaniak@gmail.com
}

received 26 May 2014, revised 15 July 2016, accepted 18 July 2016

\begin{abstract}
Identification of isomorphic kinematic chains is one of the key issues in researching the structure of mechanisms. As a result the structures which duplicate are eliminated and further research is carried out on kinematic chains that do not duplicate. This dilemma has been taken up by many scholars who have come up with a variety of ideas how to solve it. The review of the methods for identifying the isomorphism of kinematic chains suggested by researchers is contained in this study, including Hamming Number Technique, eigenvalues and eigenvectors, perimeter graphs, dividing and matching vertices. The spectrum of methods applied to the issue of identifying the isomorphism of mechanisms reflects the researchers' efforts to obtain a precise result in the shortest time possible.
\end{abstract}

Key words: Isomorphism, Structure of Planar and Spatial Kinematic Chains

\section{INTRODUCTION}

Topological methods are applied in researching the structure of mechanism. Topology is the study of those properties of objects that do not change when homeomorphisms are applied. Homeomorphism or topological isomorphism is a continuous function that has a continuous inverse function. The word 'isomorphism is used when describing the structure of mechanisms. Structural invariants are the following:

- number of links with their conjunctivity,

- number of kinematic pairs with their classes,

- joining of links of a specific conjunctivity through kinematic pairs of given classes (Conjunctivity of a link is a number of kinematic pairs that the link is composed of, which equivalently determines the number of links it can be joined with).

Fig. 1 presents an exemplary topological transformation that kinematic chains are subject to. Those kinematic chains are composed of seven links: three binary and four ternary links as well as nine kinematic pairs. A closer analysis of the figure indicates that individual links in all kinematic chains are joined together via identical kinematic pairs. For example, in all kinematic chains a kinematic pair $\mathrm{A}$ joins a binary link 1 with a ternary link 4 . Other kinematic pairs are involved in similar connections. Each kinematic chain selected for this example, is made of four loops that consist of three, four, five and six links respectively. Individual contours form the following outer loops: six-link (Fig. 1a), five-link (Fig. 1b), four-link (Fig. 1c) and three-link (Fig. 1d). In terms of structure these examples are identical or in other words isomorphic because each one of them can be obtained from the remaining ones by applying isomeric transformation in which the earlier mentioned invariants have been maintained.

To identify isomorphic structures is significant because after defining the structure of mechanisms further research is carried out for non-repeating solutions. It relates to structural synthesis in particular, whereby a sets of solutions can be obtained that fulfill specific structural formulae (Ding et al., 2011; 2012; Romaniak, 2010; 2011). a)

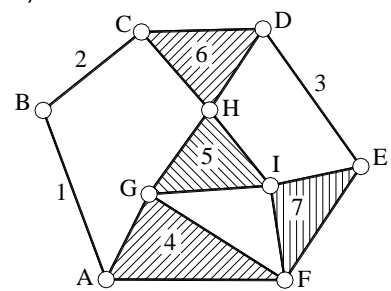

c)

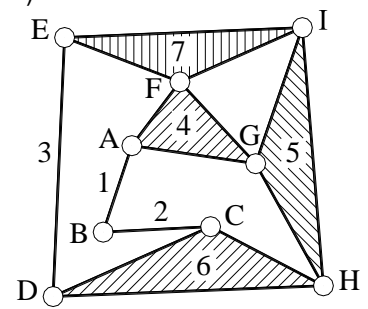

b)

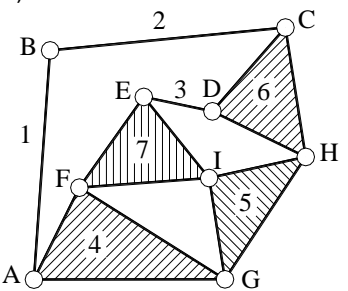

d)

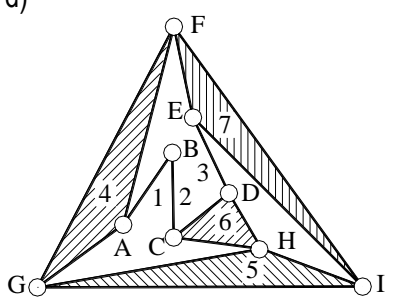

Fig. 1. Isomorphic kinematic chains

The isomorphism of kinematic chains has been taken up by many researchers who came up with a number of methods for solving this dilemma. This study discusses selected solution methods. It presents how the isomorphism of kinematic chains can be identified with the use of Hamming Number Technique (Rao and Raju, 1991), eigenvalues and eigenvectors (Chang et al., 2002; Cubillo and Wan, 2005; He et al., 2003; 2005; Uicker and Raicu, 1975), perimeter graphs (Ding and Huang, 2007; 2009), dividing and matching vertices (Zeng et al., 2014). This methods relate exclusively to the isomorphism of plane kinematic chains and do not cover spatial mechanisms. In study attempts to extend the research regarding the isomorphism of kinematic chains to the mechanisms of all groups by using new notation of spatial mechanisms. 


\section{METHODS FOR IDENTIFYING THE ISOMORPHISM OF KINEMATIC CHAINS}

The isomorphism of the three seven-link kinematic chain spatial mechanisms with 1-DOM was investigated. The set of labels for those mechanisms in group 0 includes: \{555555221, 555555311, 555554321, 555554411, 555554222, 555553331, $555553322,555544421,555544331,555544322,555543332$, 555444431, 555444422, 555444332, 555443333, 554444441, 554444432, 554444333, 544444442, 544444433, 4444444433.

Researching isomorphism required that the mechanisms were presented in a form of diagrams and structural graphs, and the adjacency matrices were determined of which elements took the value of 1 when links were adjacent and 0 when links were not adjacent. The kinematic chains were marked $C_{1}, C_{2}, C_{3}$ and structural graphs $G_{1}, G_{2}, G_{3}$ respectively.

The kinematic chains as shown were used to illustrate a few isomorphism identification methods. The first method selected is the one in which the Hamming Number Technique is applied (Rao and Raju, 1991). This method defines a specific equivalence whereby number $s_{k}=0$ is ascribed to two elements $a_{i k}, a_{j k}$ in rows $i, j$ and a column $k$ when these elements are the same $\left(a_{i k}=a_{j k}\right)$, or the number $s_{k}=a_{i k}+a_{j k}$, which is the sum of the numbers when the elements are different $\left(a_{i k} \neq a_{j k}\right)$. For example, the number $s_{1}=a_{11}+a_{21}=1$ was calculated for an adjacency matrix $M_{p 1}$ where $a_{11} \neq a_{21}$. Then the Hamming number matrix has been generated with Hamming numbers as elements obtained from the following formula:

$h_{i j}=\sum_{k=1}^{n} s_{k}$.

The $h_{12}$ element of the $H_{1}$ matrix is the sum of $s_{k}$ numbers with $k=1, \ldots, 7$ ascribed to the elements $a_{1 k}, a_{2 k}$ of the adjacency matrix $M_{p 1}\left(h_{12}=1+1+1+1+0+1+1=6\right)$.

For adjacency matrices $M_{p 1}, M_{p 2}, M_{p 3}$ the three Hamming matrices $H_{1}, H_{2}, H_{3}$ were determined respectively.

When identifying the isomorphism of kinematic chains the following definitions are in use:

- the Hamming link which corresponds to the $i$ - th link of a kinematic chain and is the sum of all elements in the $i-$ th row of the Hamming matrix $\mathrm{H}$. For example the Hamming link for the first link of a kinematic chain (see Fig.2a) is the number 28 obtained by summing up all elements from the first row of the Hamming matrix $\mathrm{H}_{1}$,

- the Hamming chain for any kinematic chain is a sum of all Hamming links. The Hamming chain for $H_{1}, H_{2}, H_{3}$ matrixes equals the number 152 ,

- the string of the Hamming link for any $i-$ th link of a kinematic chain is a string of numbers made up of the Hamming link and all the integers from 0 to $n$ in the $i$ - th row of the Hamming matrix ( $n$ is the dimension of the adjacency matrix and the Hamming matrix). For example, for the first row of the Hamming matrix $H_{1}$ a corresponding string of the Hamming link are the numbers: 28 and 13000111, where the Hamming link is 28 implying that the first row of the Hamming matrix $H_{1}$ comprises of one 7 , three $6 \mathrm{~s}$, no $5 \mathrm{~s}$, no $4 \mathrm{~s}$, no $3 \mathrm{~s}$, one 2 , one 1 and one 0 ,

- the Hamming chain string contains a Hamming chain and the Hamming chain strings in diminishing order.

$H_{1}=\left[\begin{array}{lllllll}0 & 6 & 1 & 7 & 2 & 6 & 6 \\ 6 & 0 & 5 & 1 & 4 & 2 & 0 \\ 1 & 5 & 0 & 6 & 3 & 5 & 5 \\ 7 & 1 & 6 & 0 & 5 & 1 & 1 \\ 2 & 4 & 3 & 5 & 0 & 4 & 4 \\ 6 & 2 & 5 & 1 & 4 & 0 & 2 \\ 6 & 0 & 5 & 1 & 4 & 2 & 0\end{array}\right], H_{2}=\left[\begin{array}{lllllll}0 & 6 & 4 & 3 & 5 & 4 & 6 \\ 6 & 0 & 4 & 3 & 3 & 2 & 2 \\ 4 & 4 & 0 & 5 & 3 & 4 & 2 \\ 3 & 3 & 5 & 0 & 6 & 3 & 5 \\ 5 & 3 & 3 & 6 & 0 & 3 & 1 \\ 4 & 2 & 4 & 3 & 3 & 0 & 2 \\ 6 & 2 & 2 & 5 & 1 & 2 & 0\end{array}\right]$

$$
H_{3}=\left[\begin{array}{lllllll}
0 & 6 & 4 & 3 & 6 & 5 & 4 \\
6 & 0 & 4 & 3 & 2 & 3 & 2 \\
4 & 4 & 0 & 5 & 2 & 3 & 4 \\
3 & 3 & 5 & 0 & 5 & 6 & 3 \\
6 & 2 & 2 & 5 & 0 & 1 & 2 \\
5 & 3 & 3 & 6 & 1 & 0 & 3 \\
4 & 2 & 4 & 3 & 2 & 3 & 0
\end{array}\right]
$$

The following sequences of Hamming chains have been obtained:

- matrix $H_{1} \quad 152,28,13000111, \quad 18,01110112,25$, $01301011,21,11100031$ 01110211, 18, 01110112 ,

- matrix $H_{2}$ 152, 28, 02121001, 20, 01012201, 22, 00131101, 25,01203001, 21, 01103011, 18, 00022201, 18,01100311,

- matrix $\mathrm{H}_{3}$ 152, 28, 02121001, 20, 01012201, 22, $00131101, \quad 25,01203001,18,01100311,21,01103011$ $18,00022201$.

The ultimate criteria when identifying isomorphism is a comparison of the sequences of Hamming chains. In the case of the example discussed, the sequences of Hamming chains for $\mathrm{H}_{2}$ and $H_{3}$ matrices are identical, hence the kinematic chains $C_{2}, C_{3}$ are identical in terms of structure.

Another method identifies the isomorphism of kinematic chains by applying eigensystem i.e. those kinematic chains are not isomorphic for which the eigenvalues of adjacency matrix are different (Chang et al., 2002; Cubillo and Wan, 2005; He et al., 2003; 2005; Uicker at al., 1975).

The following sequences of Hamming chains have been obtained:

- matrix $H_{1} \quad 152,28,13000111, \quad 18,01110112,25$, 01301011, 21, 11100031, 22, 00131101, 20, 01110211, 18, 01110112,

- matrix $\mathrm{H}_{2}$ 152, 28, 02121001, 20, 01012201, 22, 00131101, 25,01203001, 21, 01103011, 18, 00022201, 18,01100311 ,

- matrix $\mathrm{H}_{3}$ 152, 28, 02121001, 20, 01012201, 22, $00131101,25,01203001, \quad 18,01100311,21,01103011$, $18,00022201$.

The ultimate criteria when identifying isomorphism is a comparison of the sequences of Hamming chains. In the case of the example discussed, the sequences of Hamming chains for $\mathrm{H}_{2}$ and $\mathrm{H}_{3}$ matrices are identical, hence the kinematic chains $\mathrm{C}_{2}, \mathrm{C}_{3}$ are identical in terms of structure. 
a)

$\mathrm{C}_{1}$
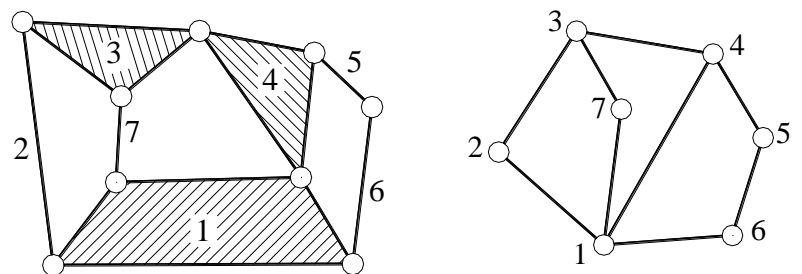

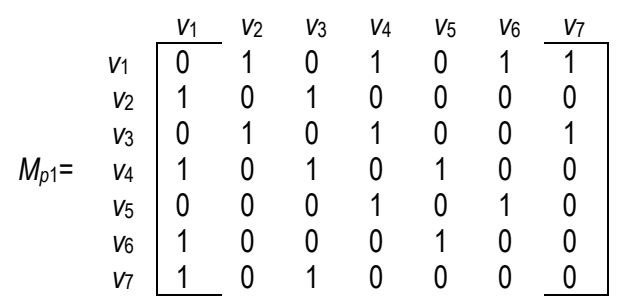

b)
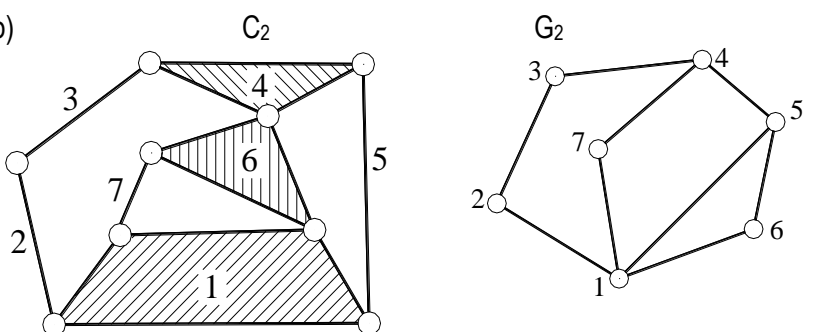

c)

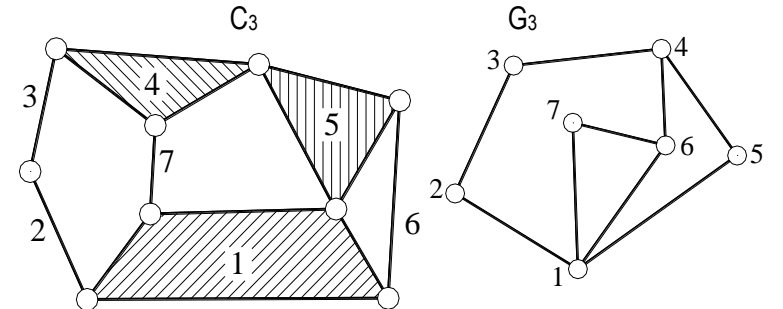

Fig. 2. Seven-link kinematic chains of mechanisms presented in a form of structural diagrams, adjacency matrices and structural graphs

The following sequences of Hamming chains have been obtained:

- matrix $H_{1} \quad 152,28,13000111, \quad 18,01110112,25$, 01301011, 21, 11100031,

01110211, 18, 01110112,

- matrix $\mathrm{H}_{2}$ 152, 28, 02121001, 20, 01012201, 22, 00131101 , 25,01203001, 21, 01103011, 18, 00022201, 18,01100311,

- matrix $H_{3} 152,28,02121001,20,01012201,22,00131101$, 25,01203001, 18, 01100311, 21, $01103011,18,00022201$. The ultimate criteria when identifying isomorphism is a comparison of the sequences of Hamming chains. In the case of the example discussed, the sequences of Hamming chains for $\mathrm{H}_{2}$ and $H_{3}$ matrices are identical, hence the kinematic chains $C_{2}, C_{3}$ are identical in terms of structure.

Another method identifies the isomorphism of kinematic chains by applying eigensystem i.e. those kinematic chains are not isomorphic for which the eigenvalues of adjacency matrix are different (Chang et al., 2002; Cubillo and Wan, 2005; He et al., 2003; 2005; Uicker at al., 1975).

For the adjacency matrix discussed (Fig.2) the following eigenvalues were obtained: $M_{p 1}: 0.441,-0.441,0,-1.178,1.178$, 2.724, 2.724, Mp2: $-1.751,-0.934,-2.165,0.252,0.708,1.157$, 2.733, Mp3: $0.708,0.252,1.157,-0.034,-1.751,-2.165,2.733$. As the eigenvalues for $C_{1}$ are different from those obtained for $C_{2}$ and $C_{3}$, the kinematic chain $C_{1}$ is not isomorphic with the $C_{2}$ and $C_{3}$ chains. To identify the isomorphism of $\mathrm{C}_{2}$ and $\mathrm{C}_{3}$ it is necessary to determine the eigenvectors. If the eigenvectors are not equivalent, the chains are structurally different. The eigenvalues and eigenvectors for $C_{2}$ and $C_{3}$ are presented in Tab. 1. The kinematic chains $C_{2}$ and $C_{3}$ have the same eigenvalues and corresponding eigenvectors, hence they are isomorphic.

Tab. 1. Eigenvalues and eigenvectors for kinematic chains $C_{2}$ and $C_{3}$

\begin{tabular}{|c|c|c|c|c|c|c|c|}
\hline \multirow[t]{10}{*}{$\mathrm{C}_{2}$} & \multicolumn{7}{|c|}{ Eigenvalues } \\
\hline & -1.751 & -0.934 & -2.165 & 0.252 & 0.708 & 1.157 & 2.733 \\
\hline & \multicolumn{7}{|c|}{ Eigenvectors } \\
\hline & 0.421 & -0.121 & 0.52 & 0.346 & 0.28 & -0.259 & 0.523 \\
\hline & -0.535 & -0.268 & -0.17 & 0.088 & 0.659 & 0.307 & 0.279 \\
\hline & 0.516 & -0.371 & -0.151 & -0.324 & 0.186 & 0.614 & 0.24 \\
\hline & -0.368 & -0.079 & 0.498 & -0.17 & -0.527 & 0.403 & 0.377 \\
\hline & 0.159 & -0.512 & -0.456 & -0.417 & -0.21 & -0.272 & 0.461 \\
\hline & -0.331 & 0.679 & -0.029 & -0.281 & 0.098 & -0.459 & 0.36 \\
\hline & -0.03 & 0.215 & -0.47 & -0.697 & -0.349 & 0.125 & 0.329 \\
\hline \multirow[t]{10}{*}{$\mathrm{C}_{3}$} & \multicolumn{7}{|c|}{ Eigenvalues } \\
\hline & 0.708 & 0.252 & 1.157 & -0.034 & -1.751 & -2.165 & 2.733 \\
\hline & \multicolumn{7}{|c|}{ Eigenvectors } \\
\hline & 0.28 & 0.346 & 0.259 & 0.121 & 0.421 & 0.52 & -0.523 \\
\hline & 0.659 & 0.088 & -0.307 & 0.268 & -0.535 & -0.17 & -0.279 \\
\hline & 0.186 & -0.324 & -0.614 & -0.371 & 0.516 & -0.151 & -0.24 \\
\hline & -0.529 & -0.17 & -0.403 & 0.079 & -0.368 & 0.498 & -0.377 \\
\hline & -0.349 & 0.697 & -0.125 & -0.215 & -0.03 & -0.47 & -0.329 \\
\hline & -0.21 & -0.417 & 0.272 & 0.512 & 0.159 & -0.456 & -0.461 \\
\hline & 0.098 & -0.281 & 0.459 & -0.697 & -0.331 & -0.029 & -0.36 \\
\hline
\end{tabular}

Graphs such as: perimeter, canonical perimeter, characteristic perimeter, together with adjacency matrices obtained for each individual graph are used by the authors of yet another kinematic chain isomorphism identifying method (Ding and Huang, 2007; 2009). The loops that form a given kinematic chain constitute the base for research. Tab. 2 presents six loops which were obtained for each kinematic chain. In further discussions the key elements are the loops with the highest number of links i.e. the longest. The kinematic chain $\mathrm{C}_{1}$ takes two loops i.e. $\mathrm{K}_{3}$ and $\mathrm{K}_{5}$, whereas kinematic chains $\mathrm{C}_{2}$ and $\mathrm{C}_{3}$ take the $\mathrm{K}_{3}$ loop. 
Tab. 2. Loops forming kinematic chains in Fig. 3

\begin{tabular}{|l|l|l|l|}
\hline Loops & \multicolumn{1}{|c|}{$\begin{array}{c}\text { Kinematic chain } \\
\mathrm{C}_{1}\end{array}$} & \multicolumn{1}{|c|}{$\begin{array}{c}\text { Kinematic chain } \\
\mathrm{C}_{2}\end{array}$} & \multicolumn{1}{|c|}{$\begin{array}{c}\text { Kinematic chain } \\
\mathrm{C}_{3}\end{array}$} \\
\hline $\mathrm{K}_{1}$ & 12371 & 123471 & 123451 \\
\hline $\mathrm{K}_{2}$ & 12341 & 123451 & 123461 \\
\hline $\mathrm{K}_{3}$ & 1234561 & 1234561 & 1234671 \\
\hline $\mathrm{K}_{4}$ & 17341 & 17451 & 1761 \\
\hline $\mathrm{K}_{5}$ & 1734561 & 174561 & 176451 \\
\hline $\mathrm{K}_{6}$ & 14561 & 1561 & 16451 \\
\hline
\end{tabular}

Perimeter graphs were formed adopting the longest loop as external. The $\mathrm{K}_{3}$ loop was selected for all kinematic chains (Fig.3).

a)
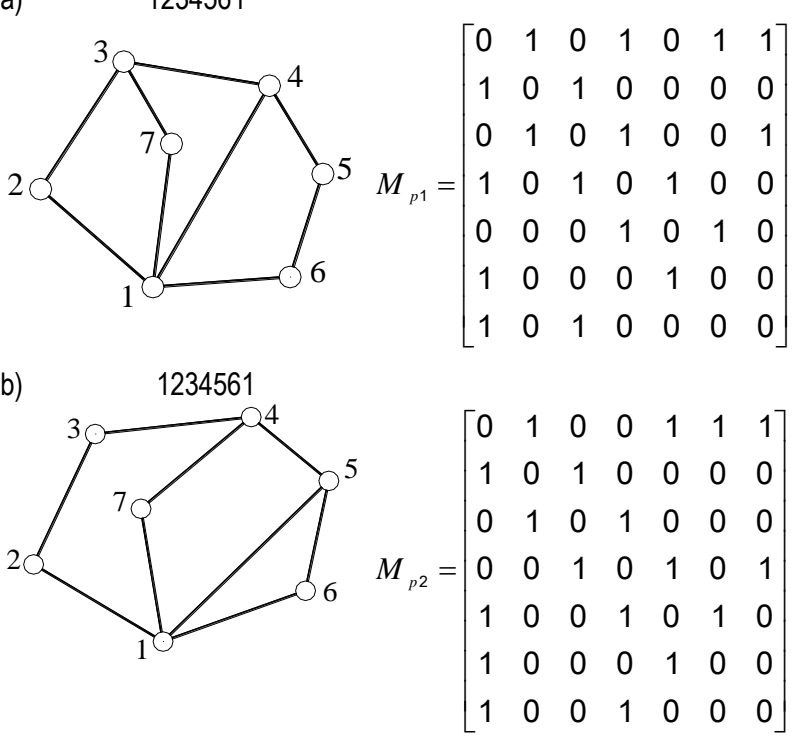

c)

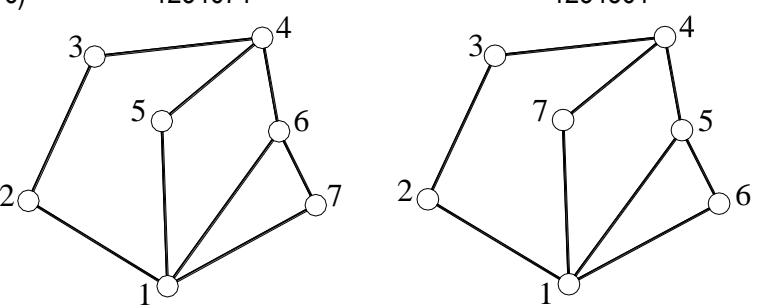

$$
M_{p 3}=\left[\begin{array}{lllllll}
0 & 1 & 0 & 0 & 1 & 1 & 1 \\
1 & 0 & 1 & 0 & 0 & 0 & 0 \\
0 & 1 & 0 & 1 & 0 & 0 & 0 \\
0 & 0 & 1 & 0 & 1 & 0 & 1 \\
1 & 0 & 0 & 1 & 0 & 1 & 0 \\
1 & 0 & 0 & 0 & 1 & 0 & 0 \\
1 & 0 & 0 & 1 & 0 & 0 & 0
\end{array}\right]
$$

Fig. 3. Structural graphs: perimeter = canonical perimeter and adjacency matrix for the $\mathrm{C}_{1}$ chain $(\mathrm{a})$, perimeter $=$ canonical perimeter and adjacency matrix for the $\mathrm{C}_{2}$ chain (b),perimeter, canonical perimeter and adjacency matrix for the $\mathrm{C}_{3}$ chain $(\mathrm{c})$

The structural graphs in Figs. 2a and $2 b$ are simultaneously perimeter graphs in Figs. $3 a$ and $3 b$. Renumbering the vertices so that each external vertex takes a consecutive integer transforms a perimeter graph into a canonical perimeter graph. By analogy, for consecutive longest loops two graphs are formed.
In the example for both loops $\mathrm{K}_{2}$ and $\mathrm{K}_{3}$ the same canonical perimeter graph was obtained. For a graph with several elements in the canonical adjacency matrix set, the matrix with the highest number of the binary string contained in the upper-right triangle of the matrix in the canonical adjacency matrix is defined as characteristic adjacency matrix, and the corresponding canonical perimeter graph is defined as characteristic perimeter graph. If for any two given graphs their characteristic perimeter graphs are identical, then the graphs are regarded as isomorphic. In the example the canonical perimeter matrices are identical for $\mathrm{C}_{2}$ and $\mathrm{C}_{3}$ hence these kinematic chains are isomorphic.

In another method for detecting the isomorphism of kinematic chains, the authors applied structural graphs and adjacency matrices (Zeng, 2014). Within an adjacency matrix the sub-blocks were selected which are defined as square sub-matrices of the adjacency matrix along the principal diagonal (Fig 4). The degrees of vertices of a graph are organised in a descending sequence making up lists denoted as List (d). If lists are different, the graphs are not isomorphic. In the case in question the degree lists for graphs $G_{1}, G_{2}, G_{3}$ are identical and form a list $\{4,3,3,2,2,2,2\}$. Each list of the List (d) constitutes the base for an adjacency matrix and the lists' vertices follow the order set up by the lists. Within those matrices, sub-blocks made by vertices of the same degree are selected (Fig. $5 a, b, c)$. Then in the sub-blocks for individual vertices a sum of squares of degrees of the vertices adjacent to them is calculated, which is called the expanded square degree of the vertex and denoted by $S$. For example, in the sub-block made up by vertices $\left\{v_{2}, v_{3}, v_{4}\right\}$ in Fig. 4 the vertex $v_{2}$ has one adjacent vertex $v_{3}$; vertex $v_{3}$ has two adjacent vertices $v_{2}$ and $v_{4}$; vertex $v_{4}$ has one adjacent vertex $v_{3}$. Thus the sum $S$ of the vertex $v_{2}$ is the square of degree of $v_{3}$ and equal to four, $S$ of $v_{3}$ is the sum of squares of the degrees of $v_{2}$ and $v_{4}$ and equal to five; $S$ of $v_{4}$ is the square of the degree of $v_{3}$ and equal to four.

In the next step a list of expanded square degrees is created, which contains the descending sequence of $S$ sums of all vertices in a sub-block and is denoted as List (S). For the sub-block $\left\{V_{2}, V_{3}, V_{4}\right\}$ in Fig.4 the list $(S)$ is $\{5,4,4\}$ and corresponds to vertices $\left\{V_{3}, V_{4}, V_{2}\right\}$.

\begin{tabular}{|c|c|c|c|c|c|c|c|}
\hline & $V_{1}$ & $v_{2}$ & $V_{3}$ & $V_{4}$ & $V_{5}$ & $V_{6}$ & $V_{7}$ \\
\hline$V_{1}$ & 0 & 1 & 0 & 1 & 0 & 1 & 1 \\
\hline$V_{2}$ & 1 & 0 & 1 & 0 & 0 & 0 & 0 \\
\hline$V_{3}$ & 0 & 1 & 0 & 1 & 0 & 0 & 1 \\
\hline$M_{p 1}=$ & 1 & 0 & 1 & 0 & 1 & 0 & 0 \\
\hline$V_{5}$ & 0 & 0 & 0 & 1 & 0 & 1 & 0 \\
\hline$V_{6}$ & 1 & 0 & 0 & 0 & 1 & 0 & 0 \\
\hline$V_{7}$ & 1 & 0 & 1 & 0 & 0 & 0 & 0 \\
\hline
\end{tabular}

Fig. 4. Exemplary sub-blocks obtained for graph $\mathrm{G}_{1}$

The equivalence of the expanded square degree lists is a necessary condition for isomorphism. For individual graphs (Fig. 5 a,b,c), the expanded square degree lists were established for three sub-blocks. The following was obtained:

- $\mathrm{G}_{1}$ : List(S) sub-block $\left\{V_{1}\right\}-\{0\}$; List(S) sub-block $\left\{V_{3}, V_{4}\right\}-$ $\{4,4\}$; List $(S)$ sub-block $\left\{V_{2}, V_{5}, V_{6}, V_{7}\right\}-\{1,1,0,0\}$ corresponding to vertices $\left\{v_{5}, V_{6}, v_{2}, v_{7}\right\}$,

- $\mathrm{G}_{2}$ : List(S) sub-block $\left\{V_{1}\right\}-\{0\}$; List(S) sub-block $\left\{V_{4}, V_{5}\right\}-$ $\{4,4\}$; List(S) sub-block $\left\{v_{2}, V_{3}, V_{6}, V_{7}\right\}-\{1,1,0,0\}$,

- $\mathrm{G}_{3}$ : List $(\mathrm{S})$ sub-block $\left\{V_{1}\right\}-\{0\}$; List $(\mathrm{S})$ sub-block $\left\{V_{4}, V_{6}\right\}-$ $\{4,4\}$; List(S) sub-block $\left\{v_{2}, V_{3}, V_{5}, V_{7}\right\}-\{1,1,0,0\}$. 
a)

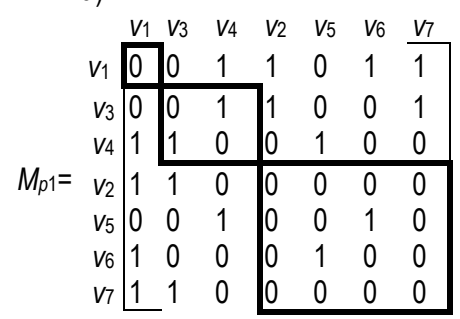

c)

\begin{tabular}{|c|c|c|c|c|c|c|c|c|}
\hline & $V_{1}$ & $V_{3}$ & $V_{4}$ & $V_{2}$ & $V_{5}$ & $V_{6}$ & $V_{7}$ \\
\hline & $V_{1}$ & 0 & 0 & 1 & 1 & 0 & 1 & 1 \\
\hline & $V_{3}$ & 0 & 0 & 1 & 0 & 1 & 1 & 0 \\
\hline & $V_{4}$ & 1 & 1 & 0 & 0 & 0 & 0 & 1 \\
\hline$M_{p 3}=$ & $v_{2}$ & 1 & 0 & 0 & 0 & 1 & 0 & 0 \\
\hline & $V_{5}$ & 0 & 1 & 0 & 1 & 0 & 0 & 0 \\
\hline & $V_{6}$ & 1 & 1 & 0 & 0 & 0 & 0 & 0 \\
\hline & $V_{7}$ & 1 & 0 & 1 & 0 & 0 & 0 & 0 \\
\hline
\end{tabular}

e) b)

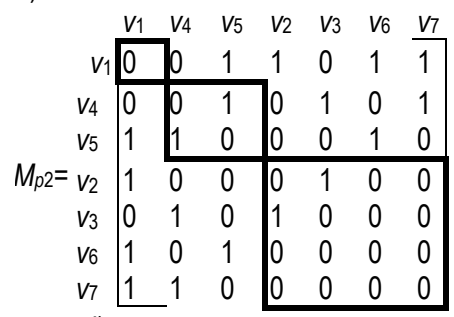

d)

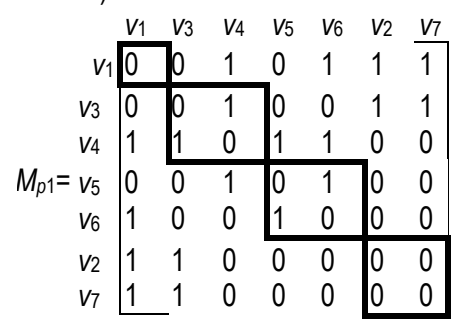

f)

\begin{tabular}{|c|c|c|c|c|c|c|c|}
\hline & $V_{1}$ & $V_{3}$ & $V_{4}$ & $V_{5}$ & $V_{6}$ & $V_{2}$ & $V_{7}$ \\
\hline$V_{1}$ & 0 & 0 & 1 & 1 & 0 & 1 & 1 \\
\hline$V_{3}$ & 0 & 0 & 1 & 0 & 1 & 1 & 0 \\
\hline$V_{4}$ & 1 & 1 & 0 & 0 & 0 & 0 & 1 \\
\hline$p 3=v_{5}$ & 1 & 0 & 0 & 0 & 1 & 0 & 0 \\
\hline$V_{6}$ & 0 & 1 & 0 & 1 & 0 & 0 & 0 \\
\hline$V_{2}$ & 1 & 1 & 0 & 0 & 0 & 0 & 0 \\
\hline$V_{7}$ & 1 & 0 & 1 & 0 & 0 & 0 & 0 \\
\hline
\end{tabular}

Fig. 5. Adjacency matrices with sub-blocks: according to List(d) for graphs $\mathrm{G}_{1}(\mathrm{a}), \mathrm{G}_{2}$ (b), $\mathrm{G}_{3}$ (c), according to List(S) for graphs $G_{1}(d), G_{2}(e), G_{3}$ (f)

According to the lists the vertices of the same expanded square degree make a sub-block (Fig. 5d,e,f). For example, after obtaining List (S), the vertices in sub-blocks in Fig.5a form subblocks in Fig.5d. Then the correlations between individual subblocks within an adjacency matrix is investigated. For this purpose the correlation degree is defined and denoted as $v_{i}\left(g_{m}\right)$. It specifies the number of vertices from the $g_{m}$ sub-block which the $v_{\mathrm{i}}$ vertex from the $g_{\mathrm{n}}$ sub-block is adjacent to. In the adjacency matrix in Fig.5d, for example, there are four sub-blocks denoted as $g_{1}, g_{2}, g_{3}, g_{4}$. The vertex $v_{4}$ in $g_{2}$ has one adjacent vertex $v_{5}$ in $g_{3}$, hence $v_{4}\left(g_{3}\right)=1$. Another correlation degree list from $g_{n}$ to $g_{m}$ is made and denoted as List $\left(g_{n}\left(g_{m}\right)\right)$ with correlation degrees $v_{i}\left(g_{m}\right)$ in descending sequence of vertices in the $g_{n}$ sub-block. The following correlation lists have been obtained for sub-blocks shown in Fig.5d, e, f:

- graph $\mathrm{G}_{1}$ : $\operatorname{List}\left(\mathrm{g}_{1}\left(\mathrm{~g}_{2}\right)\right)-\{1\}$, $\operatorname{List}\left(\mathrm{g}_{1}\left(\mathrm{~g}_{3}\right)\right)-\{1\}$, $\operatorname{List}\left(\mathrm{g}_{1}\left(\mathrm{~g}_{4}\right)\right)-\{2\}$, $\operatorname{List}\left(g_{2}\left(g_{3}\right)\right)-\{1,0\}$ corresponding to vertices $\left\{v_{4}, V_{3}\right\}$, List $\left(g_{2}\left(g_{4}\right)\right)$ - $\{2,0\}$ corresponding to vertices $V_{3}, v_{4}, \operatorname{List}\left(g_{3}\left(g_{4}\right)\right)-\{0,0\}$,

- graph $\mathrm{G}_{2}: \operatorname{List}\left(\mathrm{g}_{1}\left(\mathrm{~g}_{2}\right)\right)-\{1\}$, $\operatorname{List}\left(\mathrm{g}_{1}\left(\mathrm{~g}_{3}\right)\right)-\{1\}$, $\operatorname{List}\left(\mathrm{g}_{1}\left(\mathrm{~g}_{4}\right)\right)-\{2\}$, List $\left(g_{2}\left(g_{3}\right)\right)-\{1,0\}$ corresponding to vertices $v_{4}, v_{6}$, ( List $\left(g_{2}\left(g_{4}\right)\right)$ $-\{1,1\}$, List $\left(g_{3}\left(g_{4}\right)\right)-\{0,0\}$,

- graph $G_{3}: \operatorname{List}\left(g_{1}\left(g_{2}\right)\right)-\{1\}$, $\operatorname{List}\left(g_{1}\left(g_{3}\right)\right)-\{1\}, \operatorname{List}\left(g_{1}\left(g_{4}\right)\right)-\{2\}$, $\operatorname{List}\left(g_{2}\left(g_{3}\right)\right)-\{1,0\}$ corresponding to vertices $v_{4}, v_{5}$, List $\left(g_{2}\left(g_{4}\right)\right)$ $\{1,1\}$, List $\left(g_{3}\left(g_{4}\right)\right)-\{0,0\}$.

The equivalence of the correlation degree lists of graphs is a necessary condition for isomorphism. The correlation degree list for the $G_{1}$ graph is not equivalent with the lists for $G_{2}$ and $G_{3}$, hence the $G_{1}$ graph is not isomorphic with the $G_{2}$ and $G_{3}$.

Each vertex of the adjacency matrix of the $\mathrm{G}_{2}$ and $\mathrm{G}_{3}$ graphs belongs to the same sub-block made via the expanded square degree division and via the correlation degree division in both matrices, which means that both matrices are equal and that the $\mathrm{G}_{2}$ and $\mathrm{G}_{3}$ graphs are isomorphic.

\section{RESEARCHING THE ISOMORPHISM OF SPATIAL CHAINS}

Methods presented in Chapter II relate exclusively to the isomorphism of plane kinematic chains and do not cover spatial mechanisms. It is mainly because the base in these methods are diagrams in which kinematic pairs are noted by one symbol i.e. a circle. This is possible only for plane mechanisms whereby both the revolute and prismatic kinematic pairs are noted with such a symbol.

For spatial mechanisms the class of kinematic pairs has to be considered in their structural diagrams. As an example in the parallel mechanism presented in Fig. 6 there are four kinematic pairs of class III (The class of kinematic pairs depends on the number of parameters of kinematic bonds which a given pair imposes on the motion that one link can make in relation to the other. A link's free motion can be described by six parameters such as three revolutions and three translations along the orthogonal axis of the coordinate system. Each independent move which one link is able to make in relation to the other is called a degree of freedom W. Any constraints imposed on that movement is called a parameter of a kinematic bond $\mathrm{H}$ with values ranging from 1 to 5 . Thus $W=6-H$. Hence a kinematic pair that enables one revolution or one translation is a pair of class $\mathrm{V}$, as five out of its six degrees of freedom have been restricted. The pair of class IV means that four parameters of a kinematic bond have been imposed, the remaining two determine two degrees such as, for example, a revolution and a translation.) (three spherical pairs at the platform which make three revolutions around a coordinate axis possible and one at the base enabling two translations and one revolution), one kinematic pair of class IV (the pair at the base enabling a translation and a revolution) and one pair of class $V$ (at the base enabling one revolution). Hence a structural diagram of these mechanisms contains information about the class of kinematic pairs (Fig. 6b). a)

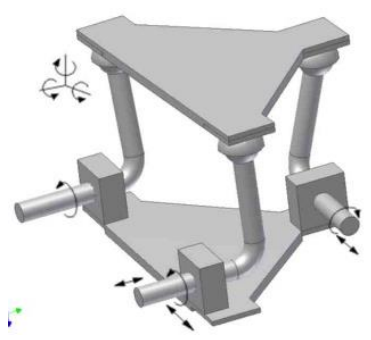

d)

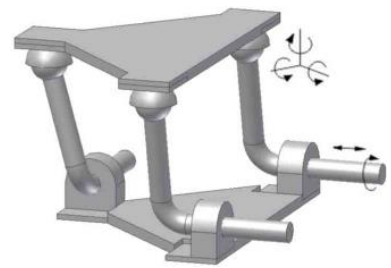

b)

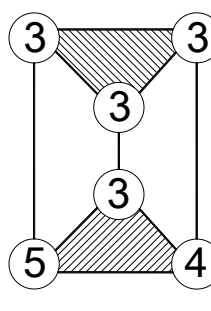

e)

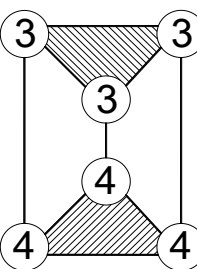

c) 543333

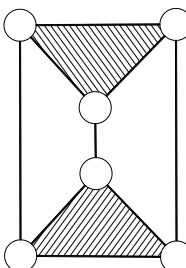

f)
Fig. 6. Parallel mechanisms $(a, d)$ in the traditional $(b, e)$ and in the generalised structural notation $(c, f)$ 
Structural analysis of spatial mechanisms indicates that the mechanisms can be grouped according to the same graphic notation in which individual mechanisms differ in the classes of kinematic pairs (Romaniak, 2010; 2011; Uicker and Raicu, 1975). For example, the parallel mechanism in Fig. $6 \mathrm{~d}$ can have a graphic representation corresponding to the parallel mechanism in Fig.6a having provided appropriate classes of kinematic pairs. A new generalised notation for spatial mechanisms has been proposed whereby classes of kinematic pairs are noted in a form of labels next to a graphic diagram (Fig. 6c, f).

Splitting the structural notation of spatial mechanisms into two parts i.e. graphic representation and a label enables the structural research used for plane mechanisms to be applied to spatial chains whose graphic diagram is identical to that of plane mechanisms. Therefore the methods of structural synthesis as well as methods for researching the chains' isomorphism, presented in Chapter II, can be used in here. Closely linked with the graphic diagram and determined following current structural formula, the labels are the sequences of numbers whose isomorphism is easy enough to check.

The proposed notation of spatial mechanisms attempts to extend the research regarding the isomorphism of kinematic chains to the mechanisms of all groups. However, it is not an ideal method as the notation in a form of a graphic diagram and a label provides a certain set of solutions restricted to various locations of kinematic pairs in a given diagram (Fig. 7). a)

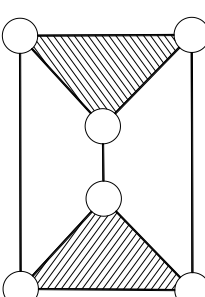

\section{3}
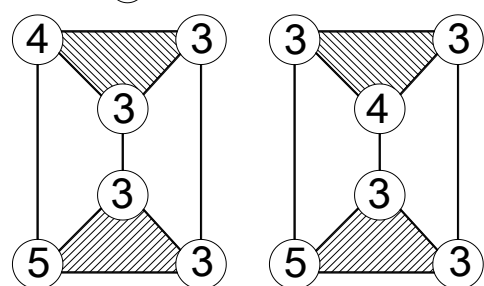

Fig. 7. A parallel mechanism with the three non-isomorphic solutions (b) obtained from the generalized notation (a)

After linking the graphic notation with labels the solutions obtained need to be checked again in order to eliminate isomorphic chains.

\section{CONCLUSIONS}

A rapidly increasing number of new methods applied to the issue of identifying the isomorphism of mechanisms reflects the researchers' efforts to obtain a precise result in the shortest time possible. In the examples presented in this paper an attempt has been made to expand the area of research to include spatial mechanisms. It has been made possible by applying a special notation for spatial chains, in which the classes of kinematic pairs are noted next to their graphic representations. Thus the research for diagrams and the labels is carried out separately, and then any method developed for flat chains can successfully be used when graphic representation is concerned. Every method discussed herein leads to an equally correct solution in terms of the isomorphism of the chains presented. Although eliminating chains of the same graphic representation, those methods do not fully sort out the problem of duplicating spatial kinematic chains. What remains to be checked is the isomorphism of chains after graphic representation has been joined with labels. Then structurally identical solutions need to be selected and eliminated.

\section{REFERENCES}

1. Chang Z. Zhang C., Yang Y., Wang Y. (2002), A New Method to Mechanism Kinematic Chain Isomorphism Identification, Mechanism and Machine Theory, 37, 411-417.

2. Cubillo J. P., Wan J. (2005), Comments on Mechanism Kinematic Chain Isomorphism Identification Using Adjacent Matrices, Mechanism and Machine Theory, 40, 131-139.

3. Ding H., Hou F., Kecskemethy A., Huang Z. (2011), Synthesis of a complete set of contracted graphs for planar non-fractionated simple-jointed kinematic chains with all possible DOFs, Mechanism and Machine Theory, 46(11), 1588-1600.

4. Ding H., Hou F., Kecskemethy A., Huang Z. (2012) Synthesis of the Whole Family of planar 1-DOF kinematic chains and Creation of Their Atlas Databases, Mechanism and Machine Theory, 47(1), 1-15.

5. Ding H., Huang Z. (2007), The Establishment of the Canonical Perimeter Topological Graph of Kinematic Chains and Isomorphism Identification, Journal of Mechanical Design, 129, 915-923.

6. Ding H., Huang Z. (2009), Isomorphism Identification of Graphs: Especially for the Graphs of Kinematic Chains, Mechanism and Machine Theory, 44, 122-139.

7. He P. R., Zhang W. J., Li Q. (2005), Some Further Development on the Eigensystem Approach for Graph Detection, Journal of the Franklin Institute, 342, 657-673.

8. He P. R., Zhang W. J., Li Q., Wu F. X. (2003) A New Method for Detection of Graph Isomorphism Based on the Quadratic Form, Journal of Mechanical Design, 125, 640-642.

9. Rao A. C, Raju D. (1991), Application of the Hamming Number Technique to Detect Isomorphism Among Kinematic Chains and Inversions, Mechanism and Machine Theory,26, 55-75.

10. Romaniak K. (2010), Generalized Methods of Kinematic Chains Structural Synthesis, International Journal of Applied Mechanics and Engineering, 15(3), 821-829.

11. Romaniak K. (2011), Methods of Structural Synthesis of Mechanisms (in Polish), Wydawnictwo ATH w Bielsku-Białej, Bielsko-Biała.

12. Romaniak K. (2011), Structural Synthesis of Parallel Mechanisms (in Polish), Modelowanie Inżynierskie, 11(42), 359-367.

13. Uicker J. J., Raicu A. (1975), A Method for the Identification Recognition of Equivalence of Kinematic Chains, Mechanism and Machine Theory, 10, 375-383.

14. Zeng K., Fan X., Dong M., Yang P. (2014), A fast algorithm for kinematic chain isomorphism identification based on dividing and matching vertices, Mechanism and Machine Theory, 72, 25-38. 